\title{
Sentinel Lymph Node Biopsy using Indocyanine Green and Blue Dye in Male Breast Cancer: A Case Report of 5 Patients
}

\section{Jun Yang}

Tongde Hospital Of Zhejiang Province

\section{Xu-Li Meng}

Zhejiang Provincial People's Hospital

\section{Yong Yu}

Tongde Hospital Of Zhejiang Province

Lei Pan

Tongde Hospital Of Zhejiang Province

\section{Jun Wu}

Tongde Hospital Of Zhejiang Province

Chang-Feng Liu ( $\triangle$ laochunteng@163.com )

Tongde Hospital Of Zhejiang Province https://orcid.org/0000-0002-5129-127X

\section{Case report}

Keywords: Male breast cancer, Sentinel lymph node biopsy, Indocyanine green, Blue dye

Posted Date: February 10th, 2021

DOl: https://doi.org/10.21203/rs.3.rs-202695/v1

License: (9) (i) This work is licensed under a Creative Commons Attribution 4.0 International License. Read Full License 


\section{Abstract}

Background: Male breast cancer is extremely rare and optimal treatments remain unclear. Most of research findings about breast cancer are derived from female patients and has not been rigorously investigated in male patients. The aim of article is to introduce the utility of indocyanine green (ICG) and blue dye mapping for sentinel lymph node (SLN) biopsy and to determine its clinical usefulness in male breast cancer.

Case presentation: A total of 5 consecutive male breast cancer patients with clinical stage ranging from I to IIA in the Tongde Hospital of Zhejiang Province during March 2013 to May 2016 were included in the present study. All patients underwent SLN biopsy with ICG and blue dye. Planed surgical resection with axillary lymph node dissection was performed. Surgically excised sentinel nodes were subjected to conventional histopathology. All of 5 cases with male breast cancer were successfully identified SLNs. Among them, 4 cases were detected by blue dye and all cases were detected by indocyanine green. A total of 10 SLNs were retrieved with an average nodal count of 2 per patient. Metastases were present in 2 nodes within 1 patients.

Conclusions: These results provide evidence that the SLN biopsy mapping with ICG and blue dye is accurate in predicting axillary lymph nodes metastases and has the potential to be a reliable criteria for staging of the axilla in patients with male breast cancer.

\section{Background}

Male breast cancer is a rare malignancy that accounts for approximately $1 \%$ of cancers in men and approximately $1 \%$ of all breast cancer worldwide[1]. Most of research findings about breast cancer are derived from female patients and has not been rigorously investigated in male patients. Mastectomy with axillary lymph node dissection has been the gold standard surgical treatment of female breast cancer. However, complications such as upper limb lymphedema and ubcutaneous hydrops are inevitable in almost a quarter of patients after extensive axillary lymph node dissection, which can have a significant impact on the patients' quality of life. To prevent unnecessary axillary lymph node dissection trauma and minimize the associated side effects, the concept of sentinel lymph node (SLN) biopsy has been proposed to provide information to the treatment of breast cancer. SLN biopsy technique has been reported to be a very accurate staging procedure in female patients with breast cancer and may further influence further therapy decisions such as extensive lymph node dissection or adjuvant medical therapy [2]. Whether it is as accurate in patients with male breast cancer has to be studied more extensively.

The use of radioisotope and blue dye for SLN mapping in breast cancer is common. Despite the documents of effectiveness and good safety data, the use of radioisotope has the potential risk of radioactive contamination, which is difficult to implement in an industrial context because of environmental and operator protection constraints[3]. Blue dye is easy to handle and cost-effective, yet it has lower detection and higher false-negative rates as some investigators maintained that the use of blue 
dye alone fail to achieve a satisfied identification rate[3]. Indocyanine green(ICG), being the only clinically recommended near-infrared imaging agent, has been demonstrated be a highly effective tracer for SLN detection. A study revealed that the SLN detection rates using ICG, blue dye or radioisotope were $99.3 \%$, $92.9 \%$ and $100 \%$ respectively[4].

Herein, we present a case of 5 patients with male breast cancer which were treated with ICG combined with blue dye mapping in SLN biopsy to determine its clinical usefulness in predicting axillary lymph nodes metastases and staging the axilla.

\section{Case Presentation}

A total of 5 consecutive male breast cancer patients aged between 61 to 72 years old with clinical stage ranging from I to IIA in the Tongde Hospital of Zhejiang Province during March 2013 to May 2016 were included in the present study. The average age of patients was 65 years old. There were 4 cases of I stage and 4 cases of IIA stage. Regarding the pathological type, majority of patients had invasive carcinoma (4 cases) with only one patient was identified to have intraductal papillary carcinoma. All of the 5 patients showed positive staining of hormone receptor. As for the Her-2 expression, 2 cases were positive and 3 were negative. The baseline characteristics of tumors were shown in table 1.

All patients underwent intraoperative SLN biopsy with ICG and blue dye. Planed surgical resection with axillary dissection were performed. Surgically excised SLN nodes were subjected to conventional histopathology. SLN mapping was performed using the Optical Molecular Imaging Operation Navigation System (MingDe Medicine, Guangzhou, China). The ICG (Dandong Pharmaceutical, Jilin, China) was dissolved to a final concentration of $1.25 \%$ from the original concentration of $2 \%$, and the methylene blue (Jumcan Pharmaceutical, Taichow, China) was diluted to a final concentration of $1 \%$.

The procedure of SLN biopsy was modified from previous studies[5]. Immediately before surgery, $1 \mathrm{ml}$ of blue dye was subcutaneously injected into the periareolar region. Five minutes later, $1 \mathrm{ml}$ of ICG solution was intradermally injected into two to four sites in the same periareolar area region. Massage was slightly performed to facilitate the movement of the tracer in the periareolar region. A hand-held fluorescence detector was used to stimulate and detect the ICG fluorescence. The lymphatic drainage was recorded and visualized on a monitor in real time(Figure 1). The fluorescent signal was followed from the injection sites to the axilla (occasionally to the intramammary region), and an incision was made to start the SLN biopsy where the fluorescence disappeared. Fluorescent lymph nodes and/or blue node were localized and excised(Figure 2). The fluorescent signal was also detected in removed nodes in vitro(Figure 3). Then, the axilla was inspected for residual fluorescent or blue nodes. Planed surgical resection with axillary dissection were performed. All removed nodes were sent for histological examination following an institutional standard protocol(Figure 4).

Transcutaneous fluorescent lymphography was visible in all individuals with male breast cancer. The extracorporeal fluorescence imaging rate reached $100 \%$. A routine incision used in dye-guided SLN biopsy in the armpit was made. When the skin and subdermal fat were incised, fluorescent lymph nodes could be 
successfully detected in 5 cases. Among them, 4 cases were detected by blue dye and all cases were detected by indocyanine green. The intracorporal fluorescence imaging rate was $100 \%$. The fluorescence signal disappeared after the visible nodes were excised. Among them, 4 cases were detected by blue dye and all cases were detected by indocyanine green.A total of 10 SLNs were retrieved, with 2 case had one SLN identified, 2 case had two, 1 case had 4 SLN identified. An average nodal count detected per patient was1.6. Two metastases was identified among the 10 retrieved SLNs $(20 \%, 2 / 10)$. No recurrence occurred within three years after the operation.

\section{Discussion And Conclusions}

Mastectomy with axillary lymph node dissection has been a common treatment for breast cancer. However, axillary lymph node dissection is associated with significant morbidity, including long-term limitations of shoulder movement, arm numbness, upper limb lymphedema and ubcutaneous hydrops, which can significantly affect the patients' quality of life[6]. To minimize the negative impact of axillary lymph node dissection, the SLN concept has been proposed to the treatment of breast cancer. SLN biopsy technique has been reported to be a very accurate staging procedure in female patients with breast cancer and may further influence further therapy decisions such as extensive lymph node dissection or adjuvant medical therapy. However, whether it is as accurate in patients with male breast cancer has to be studied more extensively. This study was designed to evaluate the clinical value of this detective method in male breast cancer.

SLN biopsy typically uses the radioisotope and blue dye. The use of radioisotopes has proven the highest detection rates, yet it is associated with some non-negligible drawbacks, including isotope handling and disposal, staff training, and legislative requirements, as well as the reluctance of patients and personnel to be exposed to radiation. Constraints of radioisotopes have raised great interest by scientists and physicians pursing the development of non-radioactive alternative methods. A number of institutions shifted to use blue dye for SLN mapping. Blue dye is easy to handle and cost-effective, yet it cannot be seen through skin or fatty tissues, and can cause allergic reactions in rare cases, even anaphylaxis[7]. Besides, the blue dye often quickly drifted away from lymph nodes, which increased the difficulties upon surgical operations. The combination of blue dye and radioisotope has been shown to be superior to either agent used separately[8]. However, this improvement lacks enough clinical significance for rating dual tracer as mandatory.

ICG, being the only clinically recommended near-infrared imaging agent, has been demonstrated be a highly effective tracer for SLN detection. As an alternative dye, ICG has numerous advantages. Firstly, it can be traced in real time in high resolution; secondly, it is cost-effective and easy to handle; third, it can be seen through skin or fatty tissues and therefore curbing the blindness in operation; last but not least, the detection rate of ICG based tracing was similar to that of radioactive agents, which make ICG a rather wise choice in SLN mapping $[9,10]$. A study compared the clinical usefulness of ICG with blue dye or radioisotope in patients undergoing SLN biopsy[4]. Data from this study revealed that the SLN detection rates using ICG, blue dye or radioisotope were $99.3 \%, 92.9 \%$ and $100 \%$ respectively. The average number 
of nodes identified by using ICG was 3.8, which was higher than that of blue dye (1.9) and radioisotope (2.0). A subsequent multi-center clinical trial replicated this finding, suggesting high accuracy of SLN mapping with ICG[11]. Though somewhat unilaterally, these studies were of great importance for demonstrating the important clinical value of the extensively explored SLN mapping with ICG and this fluorescence agent was promising for SLN mapping and axillary staging.

A study indicated that the nodal detection rate of ICG, blue dye, and ICG + blue dye samples was 97,89 , and $99.5 \%$ [12]. This result is consistent with the present study in male breast cancer. The overall SLN detection rate was $100 \%$ in this study and axillary lymph node metastases are common in this group of patients despite the clinical stages of these patients were not very advanced. It might be attributable to the relatively weak breast tissue of male patients, which make them susceptible to metastases of chest wall and axillary lymph node. The present study only contained a very limited case series, which can also affect the statistical analysis.

In this study, both extracorporeal fluorescence imaging rate and the intracorporal fluorescence imaging rate we $100 \%$, suggesting that the ICG has excellent tracing capacity in real time in high resolution. It is assumed that elder age or thicked subcutaneous fat might lead to the absent of extracorporeal fluorescence imaging in the operation, while lack of extracorporeal fluorescence sign is often caused by insufficient dosages of tracers, shortage of drainage time. Like any new surgical procedure, SLN biopsy is associated with a learning curve. This invasive procedure can be performed more effectively and safety after surgeons receive experience and accumulate skill in repeated operations. The ICG guided SLN mapping would help to train the surgical skills as this agent can be seen through skin or fatty tissue, through which the surgeons can observe the drainage of lymph nodes and improve their knowledge about the surgical operation[13]. The addition of blue dye has been shown to be superior to either agent used separately.

Many studies have shown that the ICG alone or combined with blue dye is effective in identifying SLNs in breast cancer. Majority of these studies are performed in female patients as male breast cancer is very uncommon compared to breast cancer in females. Limited studies make gender-specific findings difficult. In the present study, we provided evidence that the combination of ICG and blue dye for SLN mapping was also applicable in male breast cancer despite the false negative rate in this study was relatively high. Validation by a larger study with more patients with male breast cancer population is needed to confirm these findings.

\section{Abbreviations}

ICG: Indocyanine green; SLN:Sentinel lymph node.

\section{Declarations}

Acknowledgements 
The authors thank the patients for their participation and agreement to publication of the report.

\section{Author's contributions}

Jun Yang and Chang-Feng Liu designed research, drafted and revised the article. Lei Pan conceived the idea. Jun Yang and Xu-Li Meng collected the samples and performed most of the statistical analysis. Yong Yu and Jun Wu assisted in the follow-up work. All authors read and approved the final manuscript.

\section{Funding}

This work was supported by Medical Health Science and Technology Project of Zhejiang Provincial Health Commission(NO.2020KY500).

\section{Availability of data and materials}

The data used and/or analyzed during the current study are available from the corresponding author on reasonable request.

\section{Ethics approval and consent to participate}

This study was approved by the Ethics Committee of Tongde Hospital of Zhejiang Province. Written informed consents were obtained from all subjects.

\section{Consent for publication}

Written informed consent for publication of their clinical details and clinical images was obtained from the relative of the patient.

\section{Competing interests}

The authors declare that they have no competing interest.

\section{Author details}

1.Tongde Hospital of Zhejiang Province, Hangzhou, Zhejiang Province, China.

2.ZheJiang Provincial People's Hospital, Hangzhou, Zhejiang Province, China.

\section{References}

[1] Gucalp A, Traina TA, Eisner JR, et al. Male breast cancer: a disease distinct from female breast cancerr[J]. Breast Cancer Res Treat. 2019 Jan;173(1):37-48.

[2] Si-Qi Qiu, Guo-Jun Zhang, Liesbeth Jansen, et al. Evolution in sentinel lymph node biopsy in breast cancer. Crit Rev Oncol Hematol. 2018 Mar;123:83-94. 
[3] Houpeau JL, Chauvet MP, Guillemin F, et al. Sentinel lymph node identification using superparamagnetic iron oxide particles versus radioisotope: The French Sentimag feasibility trial. J Surg Oncol. 2016 Apr;113(5):501-7.

[4] Hojo T, Nagao T, Kikuyama M, et al. Evaluation of sentinel node biopsy by combined fluorescent and dye method and lymph flow for breast cancer[J]. Breast,2010,19(3):210-213.

[5] Papathemelis T, Jablonski E, Scharl A, et al. Sentinel Lymph Node Biopsy in Breast Cancer Patients by Means of Indocyanine Green Using the Karl Storz VITOM(R) Fluorescence Camera[J]. Biomed Res Int,2018,2018:6251468.

[6] Galimberti V, Cole BF, Viale G, Veronesi P, et al. Axillary dissection versus no axillary dissection in patients with breast cancer and sentinel-node micrometastases (IBCSG 23-01): 10-year follow-up of a randomised, controlled phase 3 trial.Lancet Oncol. 2018 Oct;19(10):1385-1393.

[7] Kang T, Yi M, Hunt K K, et al. Does blue dye contribute to success of sentinel node mapping for breast cancer?[J]. Ann Surg Oncol,2010,17 Suppl 3:280-285.

[8] He P S, Li F, Li G H, et al. The combination of blue dye and radioisotope versus radioisotope alone during sentinel lymph node biopsy for breast cancer: a systematic review[J]. BMC Cancer,2016,16:107.

[9] Aydogan F, Arikan A E, Aytac E, et al. Sentinel lymph node biopsy under fluorescent indocyanin green guidance: Initial experience[J]. Ulus Cerrahi Derg,2016,32(1):50-53.

[10] Hirche C, Murawa D, Mohr Z, et al. ICG fluorescence-guided sentinel node biopsy for axillary nodal staging in breast cancer[J]. Breast Cancer Res Treat,2010,121(2):373-378.

[11] Sugie T, Kassim K A, Takeuchi M, et al. A novel method for sentinel lymph node biopsy by indocyanine green fluorescence technique in breast cancer[J]. Cancers (Basel),2010,2(2):713-720.

[12] Guo J, Yang H, Wang S, et al. Comparison of sentinel lymph node biopsy guided by indocyanine green, blue dye, and their combination in breast cancer patients: a prospective cohort study[J]. World J Surg Oncol. 2017 Nov 2;15(1):196.

[13] Sanidas E E, de Bree E, Tsiftsis D D. How many cases are enough for accreditation in sentinel lymph node biopsy in breast cancer?[J]. Am J Surg,2003,185(3):202-210.

\section{Tables}

Table 1 Clinical features and pathological characteristics of included cases 


\begin{tabular}{|lll|}
\hline Characteristics & Case number & Percentage (\%) \\
\hline clinical stages & & \\
\hline I & 4 & 80 \\
\hline IIA & 1 & 20 \\
\hline Pathological type & & 80 \\
\hline invasive & 4 & 20 \\
\hline intraductal papillary & 1 & \\
\hline Hormone receptor & & 100 \\
\hline positive & 5 & 0 \\
\hline negative & 0 & \\
\hline HER-2 status & & 40 \\
\hline positive & 2 & 60 \\
\hline negative & 3 & \\
\hline
\end{tabular}

\section{Figures}

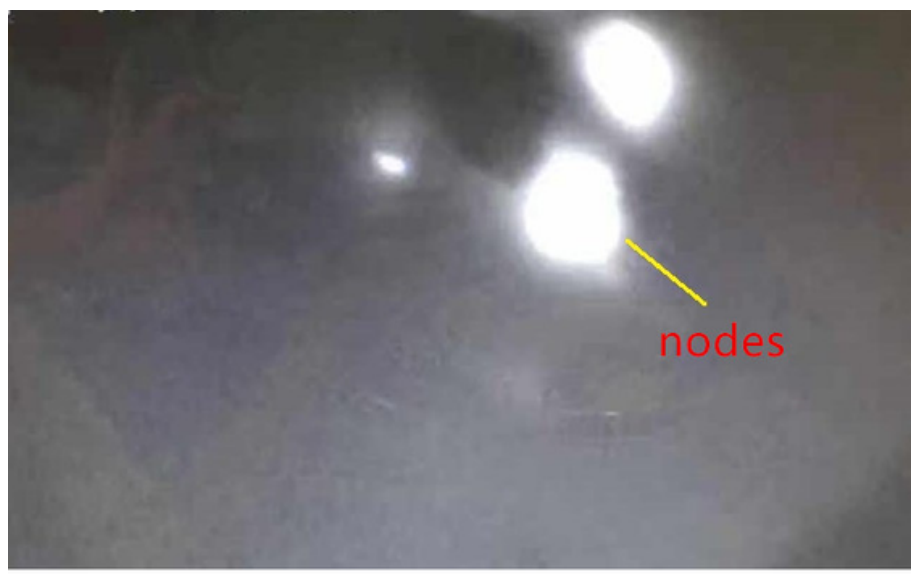

A

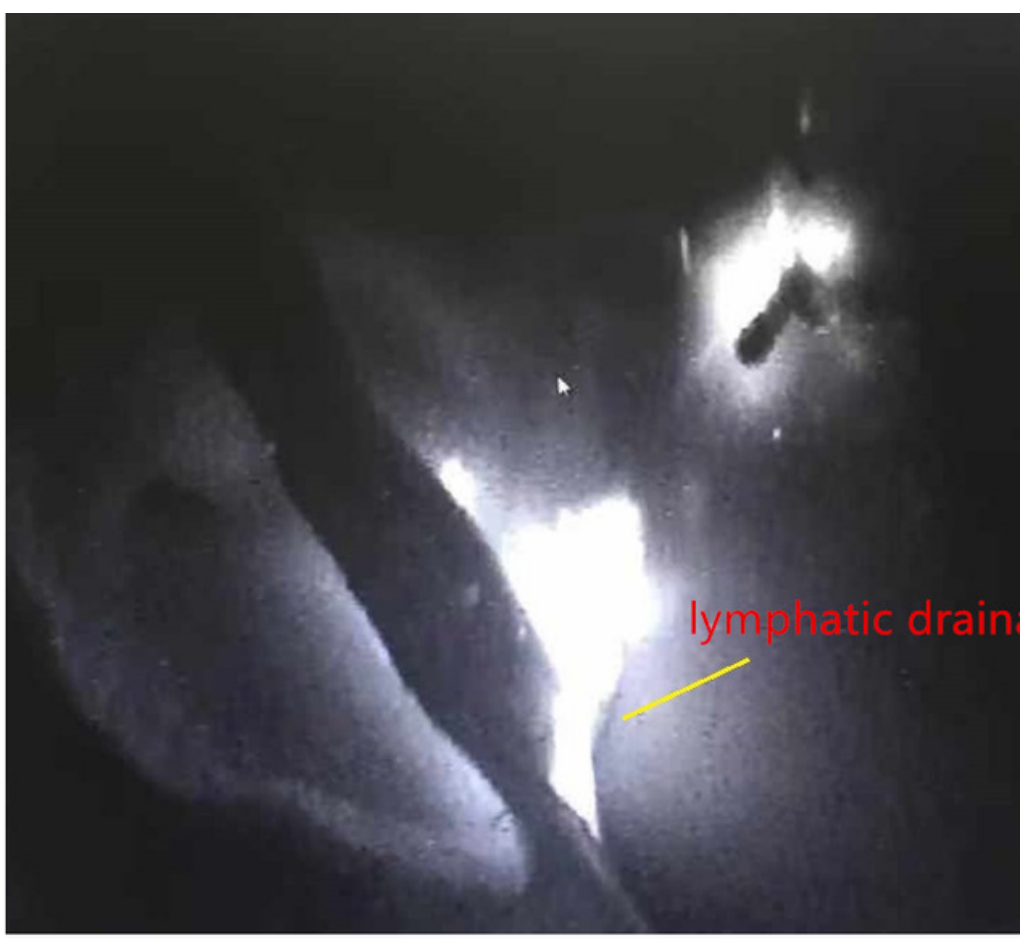

B

Figure 1 
The fluorescent signal of lymphatic drainage

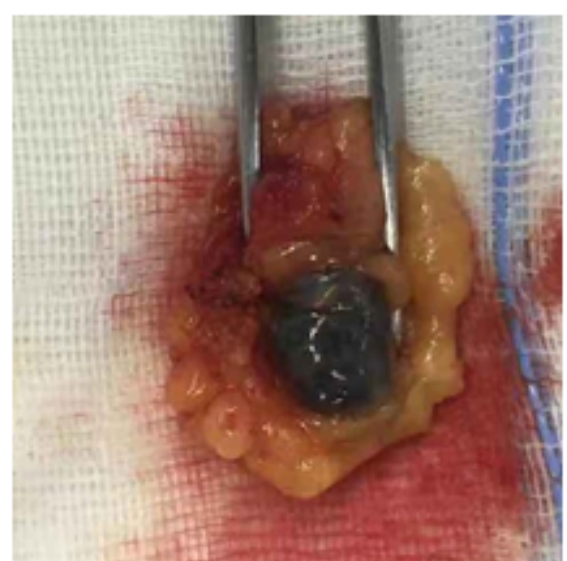

a

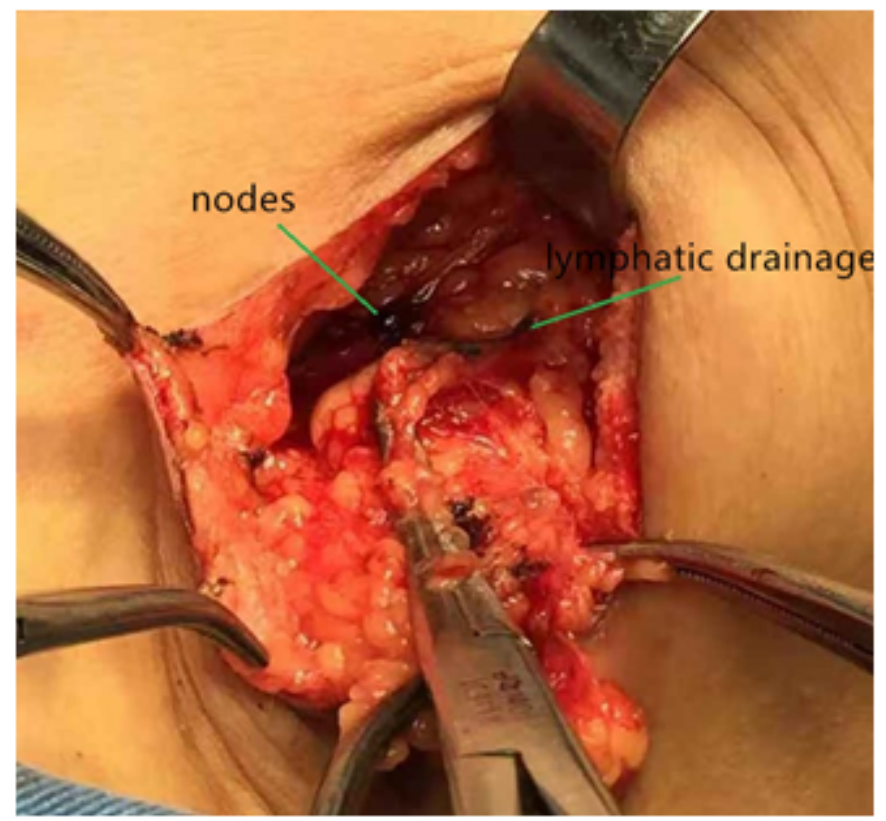

c

Figure 2

Indocyanine green and blue dye nodes

b

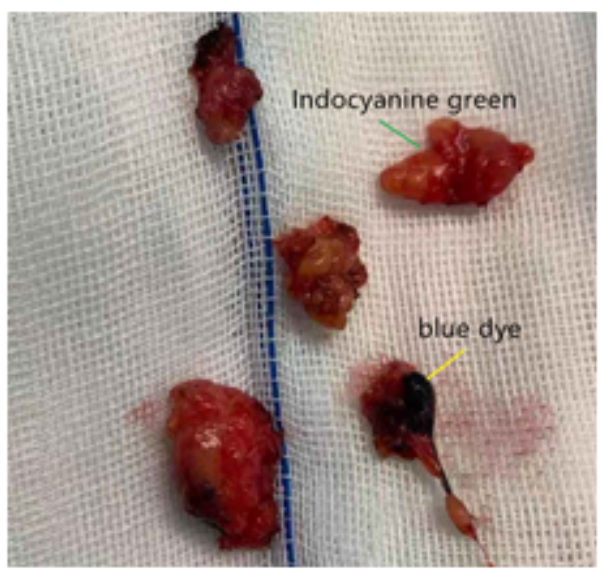




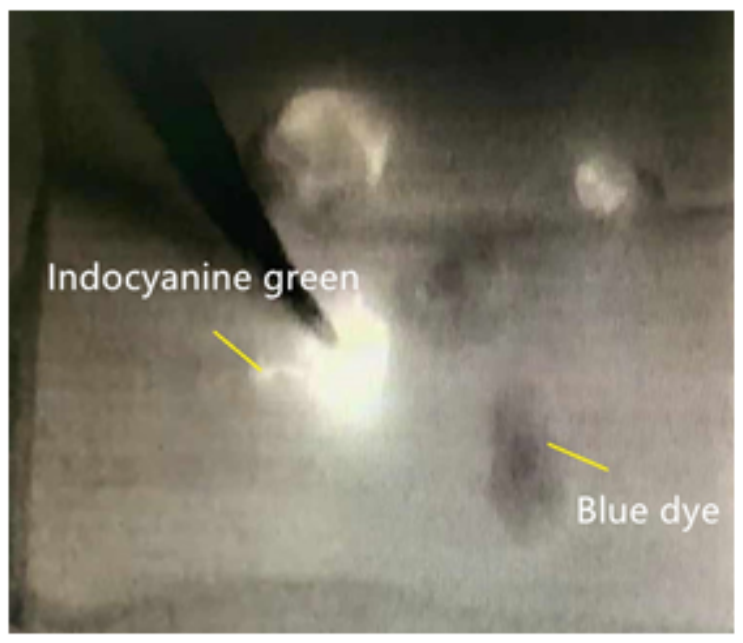

a

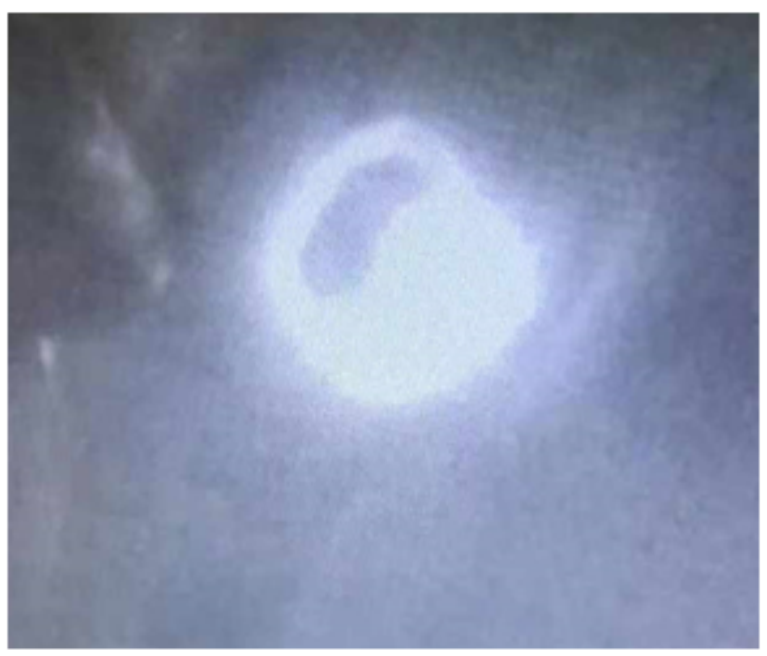

b

Figure 3

Indocyanine green and blue dye nodes The fluorescent signal was also detected in removed nodes in vitro 


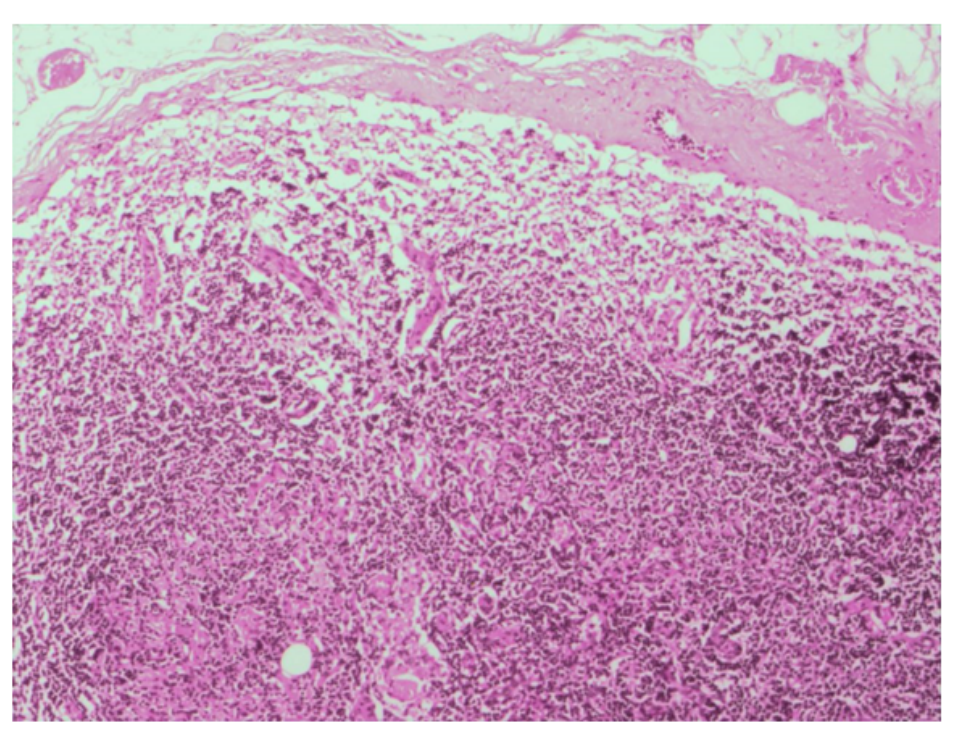

A

\section{Figure 4}

a Normal lymph nodes b Metastatic lymph node

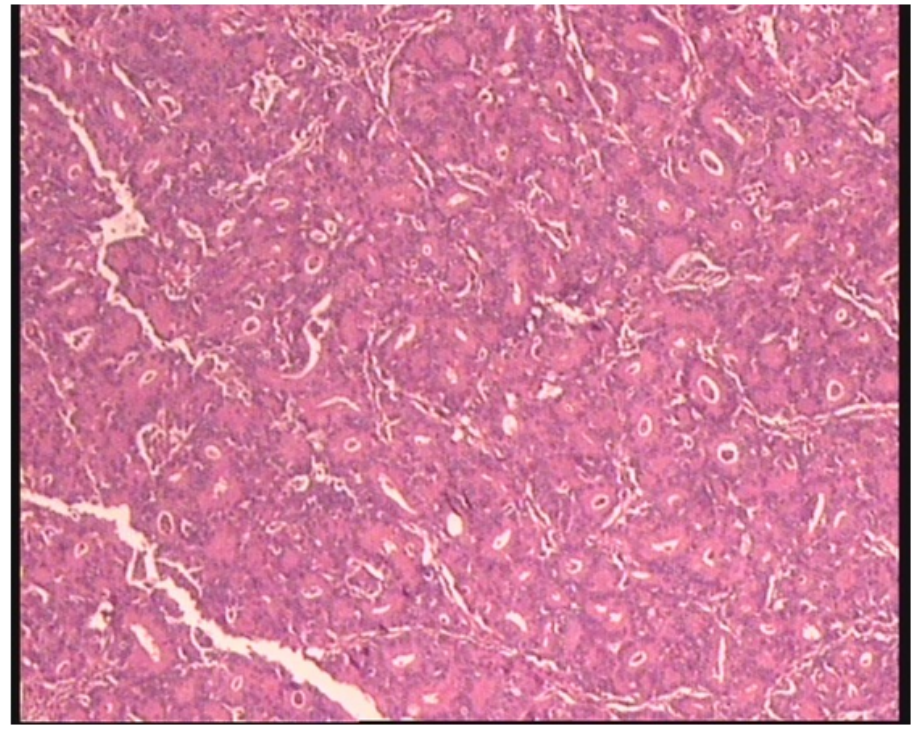

B 